\title{
Easy-implementable on-line identification method for a first-order system including a time-delay
}

\author{
Satoshi Suzuki and Katsuhisa Furuta \\ Tokyo Denki University \\ School of Science and Technology for Future Life \\ Department of Robotics and Mechatronics \\ 2-2 Kanda-Nishiki-cho Chiyoda-ku \\ Tokyo 101-8457 \\ Japan
}

\begin{abstract}
This paper proposes a simple yet effective on-line identification method for a first-order system including a time-delay. This method is based on the Laplace transformation in a real number domain and is able to estimate both coefficients of the first-order system and the time-delay simultaneously. An accuracy of the identification was investigated through a simulation. As a result, precise estimation of the method was confirmed compared to an orthodox on-line estimation technique that utilized a bilinear-model. Moreover, a guideline for a tuning of their parameters used in the method is shown. Applying the method to an actual sensor identification, issues under the practical usage were investigated, and the countermeasure was mentioned.
\end{abstract}

\section{Introduction}

Many industrial processes involve input time-delays. Control of the system including timedelays is one of important issues. For controlling systems including time-delays, Smith predictor is a practical and popular method (1). Smith proposed an idea of a predictor that compensates a time-delay effect by a feedback loop having the internal time-delay model (2). As other methods, a LQG-based control design (3), a robust stabilization control using LMI (linear matrix inequalities) (4), and an iterative identification and control method (5) were reported. And, as well as control of the system including a time-delay, an identification of processes including a time-delays is also significant. Especially for a product and for a system maintenance, a sensor diagnosis is indispensable for the industrial world, and it boils down to a problem of an identification including the time-delay. As simple solution for this issue, combination of an usual identification method and an elimination of the time-delay effect using a correlation check between the input and the output are often used. However, since this approach has a limit, other various methods have been proposed. Reed et al. applied a leastsquare algorithm to locate the cross-correlation function for the estimation of time-delay from the input / output signals (6). Teng et al. tried to estimate the time-delay of a system using the high-order numerator polynomial function (7). Teng's method has an advantage of being able to cope with an inter-sampling behavior, but this method requires sufficient long polynomial structure that can express the unknown time-delay. For dealing with the long polynomial, 
large memory and high computational power are required; hence, it is not desired for the implementation.

Additionally, the time-delay often varies with time. At chemical industrial plants, for example, a flow rate and a manipulated variable of tank reactors change by time, and these cause variations of the manipulating time-delay (8). However, from another standpoint, information of the time-delay is often useful for the comprehensive diagnosis. For instance, as the time-delay of the material flow in a chemical process can be estimated using the flow rate and length of pipes, comparison between the physically computed time-delay and the estimated time-delay from a sensor identification can raise reliability of the comprehensive system diagnosis. Therefore, several approaches that can treat unknown time-delay had been also proposed. A delay-dependent robust $H_{\infty}$ filtering for an uncertain state delay system (9) is effective as well for estimation of the state of a linear system involving time-varying parameters. The amount of the computation is, however, large; this method is inadequate for an on-line estimation because the computation requires solving of a linear matrix inequality. A neural network based approach (10) and an estimation using wavelet (11) are also known; however, their computations require also high-level arithmetic compared to the ability of the embedded computer in commercial products. Product developers have been paying many efforts to implement various functions since a computer resource of a product are restricted to reduce costs. For these practical reasons, a light program using a simple model is more preferable than a precise but complex method requiring much computation power. Additionally, field engineers who have to tune parameters of the products tend not to accept advanced concepts that are difficult to understand intuitively. For instance, a diagnosis using conventionally familiar parameters, like "gain" and "time-delay", is more popular than "singular value" or "Markov parameter". These discussions are summarized to the following requests.

- applicability to a time-varying time-delay

- easy implementation (small memory, low-computation load)

- affinity to engineers in the field

Taking these requests into consideration, a simple yet accurate identification method for the first-order system including a time-delay, real number Laplace method, is introduced in this chapter. This method utilizes the Laplace transform in order to separate the time-delay factor from a part of the system transfer function. The benefit is that preliminary information about the time-delay is not required and both target system parameters and the time-delay can be identified simultaneously. Verification of the accuracy, a guideline of the parameter tuning, and an application example are shown in later sections.

This chapter is organized as follows. Section 2 explains the real number Laplace method. Section 3 evaluates an accuracy of the method by numerical check, and leads a guideline for the parameters setting through simulation tests. In Section 4 , the method is evaluated using actual sensor response data. The discussion and conclusion are mentioned in Sections 5 and 6 , respectively.

\section{Real Numbers Laplace Identification Method}

This section proposes the identification method. Below, $\mathcal{R}$ and $\mathcal{C}$ are a class of real numbers and a class of complex numbers, respectively. The first-order system with a time-delay:

$$
G(s)=\frac{K}{1+T s} \cdot e^{-s L}
$$


is considered, where $K, T$ and $L$ are the gain, the time constant, and the time-delay, respectively. Defining the input and the output signals to the system $G(s)$ as $u(t)$ and $y(t)$, and describing their Laplace transformations as $U(s)$ and $Y(s)$, then

$$
\frac{Y(s)}{U(s)}=\frac{K}{1+T s} \cdot e^{-s L}
$$

Calculation of a natural logarithm of right- and left-hand sides of Eq. (2) yields

$$
\ln (Y(s) / U(s))=\ln K-\ln (1+T s)-s L .
$$

The Laplace transformation of $u(t)$ is described as

$$
U(s)=\int_{-\infty}^{\infty} u(t) \cdot e^{-s t} d t, \quad s \in \mathcal{C} .
$$

As $\mathcal{R} \subset \mathcal{C}$, a real number can be chosen for $s$ at Eq. (2). Therefore, assuming that $\sigma(>0, \sigma \in \mathcal{R})$ is sufficiently small number satisfying $T \sigma \simeq 0, \ln (1+T \sigma)$ in Eq. (3) can be approximated by the following Taylor expansion.

$$
\ln (1+T \sigma)=\sum_{n=1}^{\infty} \frac{(-1)^{n+1}}{n}(T \sigma)^{n}
$$

Then, Eq. (3) can be transformed as

$$
\begin{aligned}
\ln (Y(\sigma) / U(\sigma))= & \ln K-\sigma L-\ln (1+T \sigma) \\
= & \ln K-\sigma L-\left(T \sigma-\frac{T^{2}}{2} \sigma^{2}+\frac{T^{3}}{3} \sigma^{3}\right. \\
& \left.-\frac{T^{4}}{4} \sigma^{4}+\frac{T^{5}}{5} \sigma^{5} \cdots\right) \\
= & \ln K-(L+T) \sigma+\frac{T^{2}}{2} \sigma^{2}-\frac{T^{3}}{3} \sigma^{3} \\
& +\frac{T^{4}}{4} \sigma^{4}-\frac{T^{5}}{5} \sigma^{5} \cdots .
\end{aligned}
$$

Using the finite numbers of the terms in Eq. (6), the parameters of the system shown in Eq. (1) are identified through the least-square method. If these terms including from the first-order to fourth-order in the Taylor expansion shown in Eq. (6) are used, a regressor vector $\varphi$ and a parameter vector $\theta$ are decided as

$$
\begin{aligned}
\varphi(\sigma) & =\left[\begin{array}{lllll}
1 & -\sigma & \sigma^{2} & -\sigma^{3} & \sigma^{4}
\end{array}\right] \\
\theta & =\left[\begin{array}{lllll}
\ln K & L+T & \frac{T^{2}}{2} & \frac{T^{3}}{3} & \frac{T^{4}}{4}
\end{array}\right]^{T} .
\end{aligned}
$$

Then, Eq. (6) is rewritten as

$$
\varphi(\sigma) \cdot \theta=\ln \frac{Y(\sigma)}{U(\sigma)} .
$$


Next, preparing $M$ equations by substituting different real-numbers of $\sigma_{i}(>0 i=1, \cdots, M)$ into Eq. (9), those equations are summarized into the following matrix form.

$$
\begin{gathered}
{\left[\begin{array}{c}
\varphi\left(\sigma_{1}\right) \\
\varphi\left(\sigma_{2}\right) \\
\vdots \\
\varphi\left(\sigma_{M}\right)
\end{array}\right] \cdot \theta=\left[\begin{array}{c}
\ln \frac{Y\left(\sigma_{1}\right)}{U\left(\sigma_{1}\right)} \\
\ln \frac{Y\left(\sigma_{2}\right)}{U\left(\sigma_{2}\right)} \\
\vdots \\
\ln \frac{Y\left(\sigma_{M}\right)}{U\left(\sigma_{M}\right)}
\end{array}\right]} \\
\Rightarrow \Phi \cdot \theta=\Gamma
\end{gathered}
$$

Finally, an estimation of the parameter vector $\hat{\theta}$ can be obtained by a least-square method as

$$
\hat{\theta}=\left(\Phi^{T} \Phi\right)^{-1} \Phi^{T} \Gamma
$$

In Eq. (10), values of $Y\left(\sigma_{i}\right)$ and $U\left(\sigma_{i}\right)$ are computed using the Laplace transformation with their real numbers, and these values are approximated by summation of finite $N$ terms from the original Laplace transformation shown in Eq. (4). For this computing, the signal $u(t)$ is assumed to satisfy $u(t)=0$ at $t<0$, and the Laplace transformation can be approximated as

$$
\begin{aligned}
U(\sigma) & =\int_{-\infty}^{\infty} u(t) \cdot e^{-\sigma t} d t \\
& \simeq \sum_{i=1}^{N} u[i] \cdot e^{-\sigma \Delta \cdot i} \cdot \Delta
\end{aligned}
$$

where $\Delta$ is a sampling interval, and $u[i]:=u(\Delta \cdot i)(i=1, \cdots)$ is the sampled data sequence. Concerning $Y$, the approximated value:

$$
Y(\sigma) \simeq \sum_{i=1}^{N} y[i] \cdot e^{-\sigma \Delta \cdot i} \cdot \Delta
$$

is used similarly for computation of the identification.

Estimated values of $K, T$ and $L$ are extracted from $\hat{\theta}$, but the elements in $\hat{\theta}$ are redundant as shown in Eq. (8). If those elements are defined as $\hat{\theta}=:\left[\hat{\theta}_{1} \hat{\theta}_{2} \hat{\theta}_{3} \hat{\theta}_{4} \hat{\theta}_{5}\right]$, Eq. (8) gives several candidates of those parameters as follows.

$$
\begin{aligned}
\hat{K} & =e^{\hat{\theta}_{1}} \\
\hat{T}_{a} & =\left(2 \hat{\theta}_{3}\right)^{\frac{1}{2}} \\
\hat{T}_{b} & =\left(3 \hat{\theta}_{4}\right)^{\frac{1}{3}} \\
\hat{T}_{c} & =\left(4 \hat{\theta}_{5}\right)^{\frac{1}{4}} \\
\hat{L}_{a} & =\hat{\theta}_{2}-\hat{T}_{a} \\
\hat{L}_{b} & =\hat{\theta}_{2}-\hat{T}_{b} \\
\hat{L}_{c} & =\hat{\theta}_{2}-\hat{T}_{c} .
\end{aligned}
$$

In this case, there are three candidates for $T$ and $L$ as $\left\{\hat{T}_{a}, \hat{L}_{a}\right\} \sim\left\{\hat{T}_{c}, \hat{L}_{c}\right\}$. How to choose the best combination from these candidates is investigated in the next section. 


\section{Accuracy verification}

\subsection{Numerical check of computation accuracy}

The real number Laplace method utilizes approximations for the actual computation instead of the original mathematically strict descriptions. Hence, an accuracy of the identification depends on the approximation condition. In this section, effects of four factors are investigated: $\sigma$ s for the Laplace transformation, the interval $\Delta$ for the integral operation, the order of the Taylor expansion, and a buffer size $M$ for the least square method. In later discussion, a system to be identified is assumed to have intrinsic parameters as $K=1$ and $T=0.3$.

First, a relation between the approximation accuracy of $\ln (1+T \sigma)$ and the decrease ratio of the envelope curve of the integrand in the Laplace transformation was investigated. Table 1 shows the error ratio of the approximated Taylor expansions against the true value $\ln (1+T \sigma)$ for $\sigma=0.01,0.1,0.5,1,3$ and 5, respectively. The smaller percentage is interpreted as higher accurate approximation. It indicates that smaller $\sigma$ and the higher order give more precise results.

\begin{tabular}{|c|c|rrrr|}
\hline$\sigma$ & true & $\sim 1$ st term & $\sim 2$ nd & $\sim$ 3rd & $\sim 4$ th \\
\hline 0.01 & 0.003 & $-0.150 \%$ & $0.000 \%$ & $-0.000 \%$ & $0.000 \%$ \\
0.1 & 0.030 & $-1.493 \%$ & $0.030 \%$ & $-0.001 \%$ & $0.000 \%$ \\
0.5 & 0.140 & $-7.325 \%$ & $0.724 \%$ & $-0.081 \%$ & $0.010 \%$ \\
1 & 0.262 & $-14.345 \%$ & $2.807 \%$ & $-0.624 \%$ & $0.148 \%$ \\
3 & 0.642 & $-40.219 \%$ & $22.880 \%$ & $-14.979 \%$ & $10.575 \%$ \\
5 & 0.916 & $-63.704 \%$ & $59.074 \%$ & $-63.704 \%$ & $74.421 \%$ \\
\hline
\end{tabular}

Table 1. Error ratios of Taylor expansions approximated using different $\sigma$

Next, an effect of the finite summation instead of the infinite integration was investigated. Table 2 shows values of the envelope function $e^{-\sigma T_{F}}$, that appears in the integrand shown in Eq. (4), against different combinations of $\sigma$ and $T_{F}$. The smaller value shows that the rounding error at the end point of the integral computation is small; hence, the approximation is close to the true value.

\begin{tabular}{|c|rrrrr|}
\hline$\sigma$ & $T_{F}=3$ & $T_{F}=5$ & $T_{F}=10$ & $T_{F}=20$ & $T_{F}=30$ \\
\hline 0.01 & 0.9704 & 0.9512 & 0.9048 & 0.8187 & 0.7408 \\
0.1 & 0.7408 & 0.6065 & 0.3679 & 0.1353 & 0.0498 \\
0.5 & 0.2231 & 0.0821 & 0.0067 & $\underline{0.0000}$ & 0.0000 \\
1 & 0.0498 & 0.0067 & 0.0000 & 0.0000 & 0.0000 \\
3 & 0.0001 & 0.0000 & 0.0000 & 0.0000 & 0.0000 \\
5 & 0.0000 & 0.0000 & 0.0000 & 0.0000 & 0.0000 \\
\hline
\end{tabular}

Table 2. Attenuation rates of the envelope function $e^{-\sigma T_{f}}$

Table 1 shows that the approximation with the smaller $\sigma$ is better even if the order of the Taylor expansion is small. Meanwhile, table 2 indicates that the summation computation by the small $\sigma$ cannot cover the integrated area of the original integral calculation sufficiently even if the integral time is long. That is, there is a trade-off in choice of $\sigma$ due to the truncation error at the finite-time approximation of the Laplace transformation. Consideration of these tables suggests the following guide-line for the selection of $\sigma$ and $T_{F}$ : 
- $\sigma=0.5$ is adequate for the real number Laplace transform computation.

- More than third-order approximation with small $\sigma$ that is less than 0.5 is necessary for the Taylor expansion approximation ${ }^{1}$.

- $T_{F}=20[s]$ appears to be adequate as the integral time ${ }^{2}$ for the approximation.

Aforementioned investigation surmises the following remarks.

\section{Remarks}

- $\sigma$ has to be chosen as small as possible in order to satisfy $T \sigma \simeq 0$ for good approximation of the logarithm function in the Taylor expansion. Furthermore, the measured data has to be long so as to attenuate an integrand of the Laplace transformation.

- For a target system including a large time-constant, it is necessary to choose small $\sigma$ or to extend the integral interval by same reason of the above item.

- High order polynomial in the Taylor expansion gives more accurate approximation; however, this increases the size of the regressor and the amount of the computation.

\subsection{Simulation verification using test signal}

Accuracy of the real number Laplace method was investigated using the sample response data from a virtual target system when an input signal of a pulse wave was added to the system. The amplitude and the cyclic period of the pulse input were chosen as 0.5 and 2 seconds. It was assumed that the target system had a gain of $K=1$, a time-constant of $T=0.3$, and a time-delay of $L=0.1[s]$. Parameters for the identification were chosen as follows based on the remarks mentioned in Section 3.1.

- the range of real numbers for the Laplace transformation: $\sigma=0.5 \sim 0.7$ at 0.01 interval

- the number of points for the least-square method: $M=20$

- the number of points for numerical integral: $N=2000$

- sampling time: $\Delta=10[\mathrm{~ms}]$

These conditions leads $T_{F}=\Delta t \times N=20[s]$. First, effects of the finite truncation of the Taylor expansion for $\ln (1+T \sigma)$ was inspected by changing the "Maximum Order of truncation Terms"(MOT) in the Taylor series. Table 3 shows the results. Note that the number of candidates of pair $\{T, L\}$ increases as the truncation order increases because of a redundancy in their parameter vectors. The result shows that the identified parameters were fairly close to their true values. Not only the gain and time-constant but also the time-delay could be estimated. Table 3 shows also that the first pair $\left\{\hat{T}_{a}, \hat{L}_{a}\right\}$ of the higher order truncation leads more accurate result.

Last check is about an effect of the integral interval $T_{F}$ of the Laplace transformation approximation. Results for two cases of $T_{F}=10$ and $T_{F}=40$ are summarized in Tables 4 and 5, respectively. The identified parameters for $T_{F}=10$ (Table 4) are wholly inferior to the case of $T_{F}=20$ (Table 3). This is, of course, because a finite time interval computation was used instead of an infinite interval in the integral computation. Especially, the case of MOT=4 gave wrong result since pairs of $\left\{\hat{T}_{b}, \hat{L}_{b}\right\}$ and $\left\{\hat{T}_{c}, \hat{L}_{c}\right\}$ were complex numbers. On the other hand, the case of $T_{F}=40$, as shown in Table 5, improved the results slightly compared to the case of $T_{F}=20$. The 40 seconds appears, however, excess for $T_{F}$ since twice-time computing is needed. Hence, it appears that $T_{F}=20$ is adequate to the present sample system.

\footnotetext{
1 The percentage of the approximation error is small at -0.0809 [\%] in this case. (From Table 1)

2 The remainder of the integrated area is tiny at 0.0000 [\%]. (From Table 2)
} 


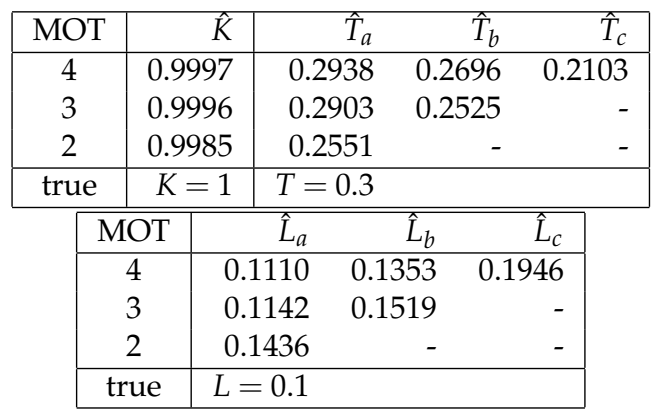

Table 3. Identification results using test signal with $T_{F}=20$

\begin{tabular}{|c|r|rrr|}
\hline MOT & $\hat{K}$ & $\hat{T}_{a}$ & $\hat{T}_{b}$ & $\hat{T}_{c}$ \\
\hline 4 & 0.9974 & 0.2080 & $0.1667+0.2888 \mathrm{i}$ & $0.2772+0.2772 \mathrm{i}$ \\
3 & 0.9981 & 0.2612 & 0.1717 & - \\
2 & 0.9977 & 0.2494 & - & - \\
\hline \multirow{4}{*}{ MOT } & $\hat{L}_{a}$ & $\hat{L}_{b}$ & $\hat{L}_{c}$ \\
\cline { 2 - 5 } & 4 & 0.1856 & $0.2268-0.2888 \mathrm{i}$ & $0.1164-0.2772 \mathrm{i}$ \\
& 3 & 0.1372 & 0.2268 & - \\
& 2 & 0.1473 & - & - \\
\cline { 2 - 5 } & & & &
\end{tabular}

Table 4. Identification results using test signal with $T_{F}=10(M=1000)$

\subsection{Comparison with a bilinear-model method}

In this section, the real number Laplace method is compared to other conventional identification method able to be implemented on-line to show the effectiveness. Here, a recursive leastsquare method with a discrete model was chosen as the conventional method. This method utilizes a bilinear transformation, and is termed bilinear-model method simply later. Details of the bilinear-model method are described in Appendix. A. Same test signal that includes no timedelay was applied to the bilinear-model method. Figure 1 indicates five cases of transitions of the estimated values of time-constant $T$ and gain $K$. Each graph was obtained by changing the DF. The DF were specified as 1,5,10,20 and 40, and these values correspond to sampling intervals $0.01,0.05,0.1,0.2$ and $0.4[s]$, respectively. From the figure, $D F=5(d t=0.05)$ appears a best condition since the identified parameters were close to their true values $(T=0.3, K=1.0)$. Conversely, as shown in this analysis, the recursive type of identification methods requires selection of the adequate DF. The DF reportedly has to be chosen considering the time-constant of the target system. For the sensor diagnosis, however, the time-constant itself changes across the ages; hence, this is one of the drawbacks. In contrast, the proposed real number Laplace method is applicable to a change of a time-constant in the target system.

Next, the bilinear-model method with $D F=5$, that was the best tune for the decimation, was applied to the test signal including a time-delay. Simulation tests were executed against different values of the time-delay. The results are illustrated in Fig. 2 . The identified parameters of $K$ and $T$ became larger than the true values as the time-delay increased. Since the bilinear-model method does not have an ability of the time-delay estimation, other time-delay estimator, such as a correlation analysis between the input and output signal, is required. However, if the 


\begin{tabular}{|c|c|c|c|c|c|c|c|c|}
\hline MOT & \multicolumn{2}{|c|}{$\hat{K}$} & \multicolumn{2}{|r|}{$\hat{T}_{a}$} & \multicolumn{2}{|r|}{$\hat{T}_{b}$} & \multicolumn{2}{|c|}{$\hat{T}_{\mathcal{C}}$} \\
\hline 4 & \multicolumn{2}{|c|}{0.9998} & \multicolumn{2}{|c|}{0.2986} & 0.2 & & \\
\hline 3 & \multicolumn{2}{|c|}{0.9996} & \multicolumn{2}{|c|}{0.2911} & 0.2 & & \multicolumn{2}{|c|}{$\begin{array}{r}0.2344 \\
-\end{array}$} \\
\hline 2 & \multicolumn{2}{|c|}{0.9985} & \multicolumn{2}{|c|}{0.2552} & & 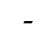 & \multirow[b]{2}{*}{$\hat{L}_{c}$} & . \\
\hline \multicolumn{2}{|c|}{ MOT } & & $\hat{L}_{a}$ & & $\overline{\overline{L_{b}}}$ & & & \\
\hline \multicolumn{2}{|c|}{4} & \multirow{2}{*}{\multicolumn{2}{|c|}{0.1068}} & 0.1 & & \multicolumn{2}{|c|}{0.1510} & \\
\hline \multicolumn{2}{|c|}{3} & & & 0.1 & & & - & \\
\hline & & \multicolumn{2}{|c|}{0.1436} & & - & & - & \\
\hline
\end{tabular}

Table 5. Identification results using test signal with $T_{F}=40(M=4000)$

time-delay effect is removed insufficiently, the estimation becomes worse. Figure 2 highlights this weak point of the bilinear-model method . Conversely, from an insufficient results given by the bilinear-model method, it can be concluded that the proposed real number Laplace method is superior in terms of an estimation accuracy and of robustness against a time-delay.

\section{Application-level verification}

In this section, the proposed identification method was verified using an actual measured data. Here, a sensor in the engine control system was chosen for an example. For not only the performance retention but also an environmental conservation, a sensor is significant for the engine control. Response anomaly of a degraded sensor induces a change of the timeconstant or the inaccurate gain; hence, it is relatively easy to determine the likelihood of the degrading by checking the step response in case of an unit testing. Rotational speed of the engine, however, varies awfully depending on various factors such as driver's demand and the load condition, and the time-delay also varies (12). Since the proposed real number Laplace method can be applied to unknown time-delay, this example is adequate for the verification.

\section{1 $\mathrm{O}_{2}$ sensor in an engine system}

The $\mathrm{O}_{2}$ sensor that is treated here monitors an oxygen density in combustion gas near the engine cylinder at an exhaust pipe. Air-fuel ratio (AFR) is computed based on the measured $\mathrm{O}_{2}$ density, and the input signal for the sensor identification is assumed to be a AFR of the fuel gas. The AFR of the fuel gas varies mainly depending on an intake air mass and the amount of fuel consumption from the injector. All of the injected fuel, however, does not evaporate into air, and portion of the fuel adheres to a wall of the pipe. Further, the gas is transported via the four-stroke cycle: Intake, Compression, Combustion, and Exhaust. Because of these processing, the delay from the injection to the detection at the $\mathrm{O}_{2}$ sensor is generated and changes dynamically.

In order to apply the proposed method to the $\mathrm{O}_{2}$ sensor identification, variation of the fuel gas AFR was chosen for the input signal $u(t)$, and the other AFR of the exhaust gas monitored by the sensor was used as the output signal $y(t)$. The transmission lag at the pipe and the engine cyclic processing were treated as one delay element. Supposed that the dynamics can be modeled with a first-order system, the whole of the transfer function can be treated as Eq. (1). In an engine control, small additional operation is permitted during the constant speeds. So-called "active excitation", that changes the input AFR by small amplitude square wave, is executed in a product car. Referring an actual case, a square wave whose amplitude and cyclic 

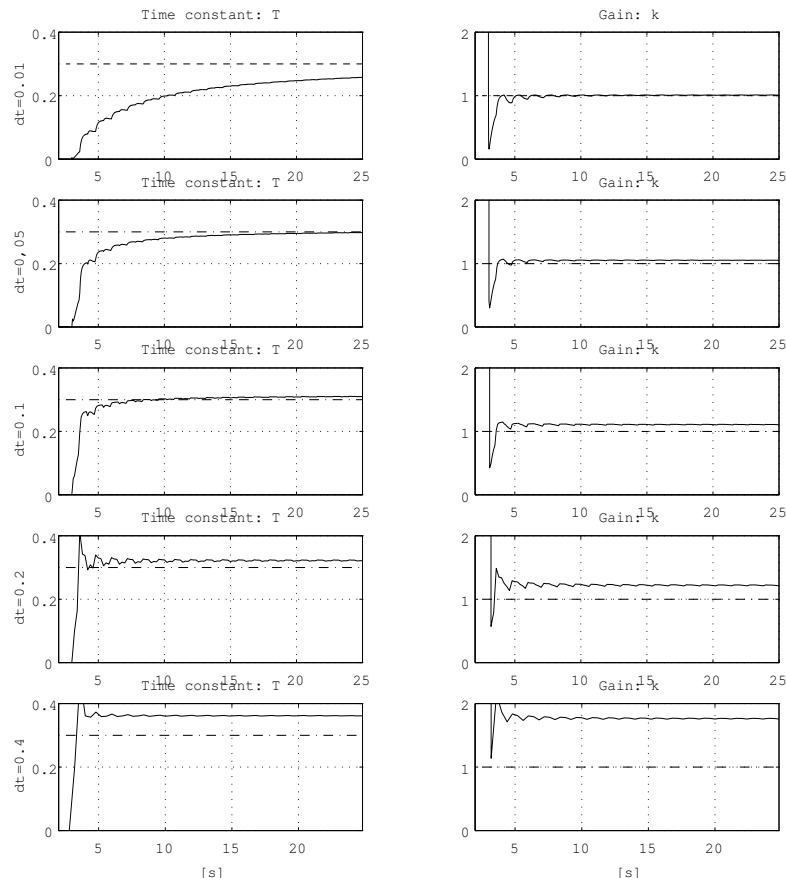

Fig. 1. Transitions of identified parameters by the bilinear-model method ( $\mathrm{dt}$ is the sampling interval after decimation.) Dashed lines denote the true values $\bar{T}=0.3$ and $\bar{k}=1$.

period were $\pm 0.5[A F R]$ and $1.32[s]$ was used. Parameters for the identification were chosen as follows based on aforementioned guideline.

- the order to approximate the Taylor expansion: third order

- the range of real numbers for the Laplace transformation: $\sigma=0.5 \sim 0.7$ at 0.01 interval

- the number of points for the least-square method: $M=20$

- the number of points for numerical integral: $N=2400$

Sampling time $\Delta=8.2[\mathrm{~ms}]$ was decided by the measurement condition. These conditions leads $T_{F}=\Delta \times N \simeq 20[s]$.

\subsection{Verification using actual data}

The data was obtained using a bench test with a four-cycle 2.3 liter engine. At first, the data measured at a speed of constant $80[\mathrm{~km} / \mathrm{h}]$ was filtered through a LPF $1 /(1+10 \mathrm{~s})$ to eliminate the bias, and the step response crossing the zero-level was obtained. The identification result is shown at the case- 1 in the Table 6 . Contrary to the present authors' expectation, the identified values were complex numbers and the correct ones were not obtained. To find the reason, values of terms in equations were checked. As a result, it was confirmed that the integration value in the transformation (13) was smaller than other case that was computed using an ideal wave form. The zero-crossing signal tends to be affected by the noise; hence, the actual signal appears ill-conditioned involving small $\mathrm{S} / \mathrm{N}$ ratio. To avoid this issue, both the input and the 

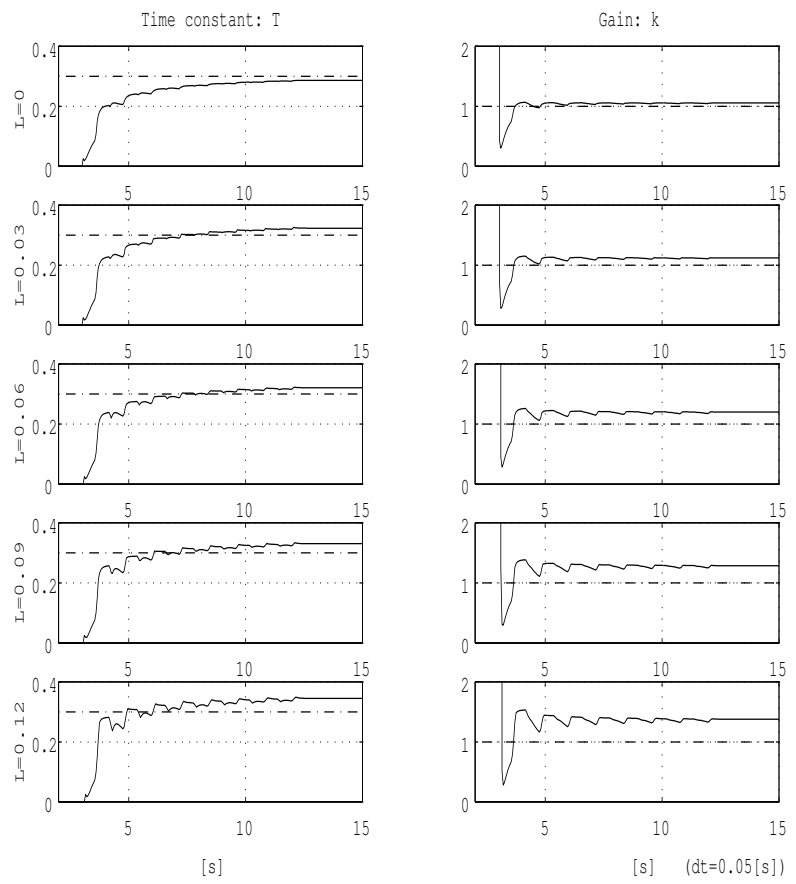

Fig. 2. Transitions of identified parameters by bilinear-model method : Comparison against different time-delay.

output signals were modified by adding 0.5 to them so as to be greater than 0 , as shown in Fig. 4. The result of an identification using the modified signals is shown at the case- 2 in Table 6. The identified gain $\hat{K}$ is close to the true value, and real number time-constant value $\hat{T}_{a}$ was obtained. Accuracy of the identification was improved, but still insufficient.

Considering of a mathematical property of Eq. (13) again, it was surmised that an initial value of the integration period affected strongly the computation since a value of the envelope curve $e^{-\sigma t}$ is large around $t \simeq 0$. Upper graph in Fig. 5 is an enlargement of the initial rising of the signals shown in Fig. 4. As indicated with an arrow in the graph, a residual vibration of a previous step-response remained in the interval of $t=8.19 \sim 8.5$, and it was found that $y \simeq 0$ was not satisfied at the same period. To remove this adverse effect, the integration period was modified so as to be satisfied with $u \simeq 0$ and $y \simeq 0$. In short, the data was shifted in time direction by 30 sampling points to change the initial time from $t=8.13$ to $t=8.44$. The modified signals are shown at the lower graph in Fig. 5. Using the modified signals, the identification result was improved as written at the case- 3 in Table 6 . The identified gain $\hat{K}$, the time-constant $\hat{T}_{a}$, and the time-delay $\hat{L}_{a}$ were sufficiently close to their true values. Though the second candidates of the time-constant and the time-delay were complex numbers, the identification-method worked well because it was already confirmed that the proposed method lead accurate values in the first candidate term from the redundant ones as mentioned in Section 3.2. 


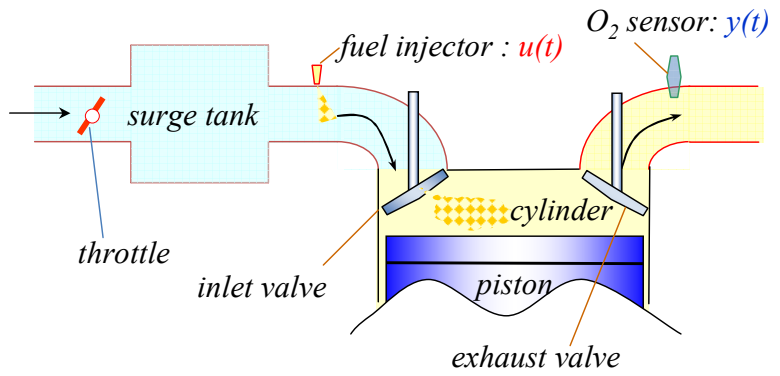

Fig. 3. $\mathrm{O}_{2}$ sensor in the engine system

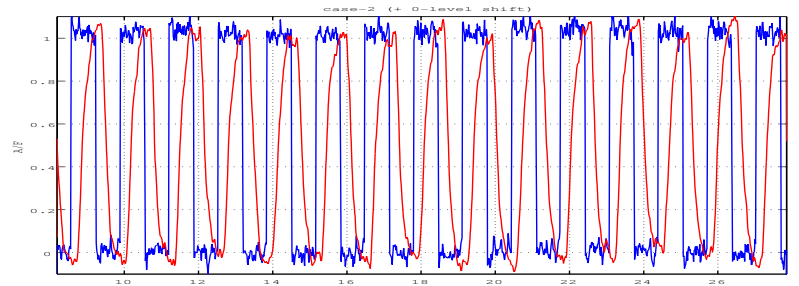

Fig. 4. Fluctuation component of the excitation (blue), and the modified sensor output (red).

For confirmation, using the original measured input signal AFR, simulation responses were computed through two models: a model with identified parameters (of the case- 3 in Table 6), and a model with nominal parameters (of the "true" in the same table). The resultant wave-forms are shown in Fig. 6. The original raw experimental signal was also drawn in each graph. The graphs illustrate that the time-delay was estimated correctly by comparing those timings of the rising response. The amplitude of the response computed with the identified parameters appears slightly small. However, slant angles of the rising curve of both response data are same; hence, it can be accepted that the estimated time-constant was correct.

\subsection{Investigation of effect by the excitation signal condition}

Aforementioned section showed that an accuracy of the identification became low if the starting point was not chosen adequately or a shifting of the input/output signal to the positivevalue zone was insufficient. Since it is better to know degree of the modification as a guideline for the implementation, the sensitivity analysis is mentioned in this section. Normative response signal was computed through the simulation by adding a rectangular excitation signal (amplitude $=1$, period=1.32 [s], sampling interval=8.2 [ms]) to the transfer function whose parameters were same as the former system $(K=1.0, T=0.18, L=0.17)$. Next, using test signals that were obtained by changing the delay of the excitation timing $b[s]$, and the vertical shift from the nominal response signal $a$, as shown in Fig. 7, the difference of the identified results were investigated. The $a$ is a ratio of the part that is below a zero-level, and the signal varies from 0 to 1 when $a=0$. 

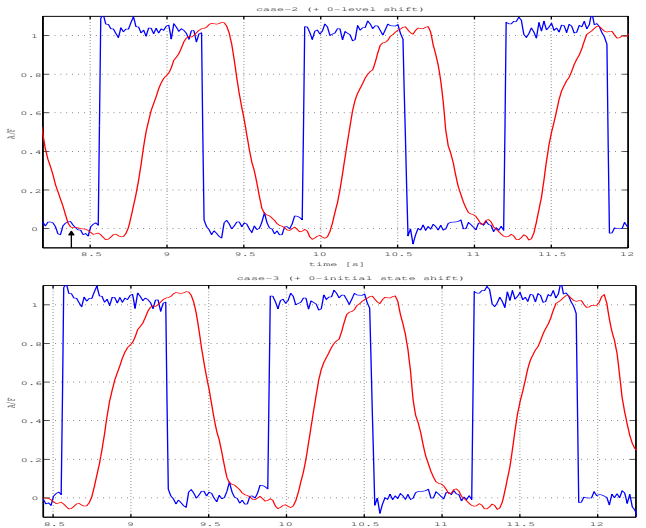

Fig. 5. Magnified graph of input and output signals (upper), and the signals modified of the initial points (lower)

\begin{tabular}{|c|r|rc|rc|}
\hline case & $\hat{K}$ & $\hat{T}_{a}$ & $\hat{T}_{b}$ & $\hat{L}_{a}$ & $\hat{L}_{b}$ \\
\hline 1 & c.n. & c.n. & c.n. & c.n. & c.n. \\
2 & 0.94 & 0.28 & c.n. & -0.0045 & c.n. \\
3 & 0.94 & 0.16 & c.n. & 0.20 & c.n. \\
\hline true & $K=1.0$ & $T=0.18$ & & $L=0.17$ & \\
\hline
\end{tabular}

Table 6. Comparison of the identified results

First, $b$ was fixed as $b=0$, and the identification accuracies against different ratios of positive zone in vertical direction were computed. The results are summarized in Table 7 . Though the gain $K$ was estimated correctly for $a=0 \sim 0.5$, the time-constant $T$ and time-delay $L$ became worse gradually. For the $a$ over 0.5 , their results rapidly got worse. These results show that the signal including much positive values is better for the correct identification. It appears that the tolerance is $a=0.0 \sim 0.2$.

Next, $a$ was fixed as $a=0$, the identification accuracies against different delays at start of the excitation were investigated similarly. The results is Table 8. Their gains were identified with similar accuracy to the former case. The accuracies of the time-constant and the timedelay became worse as $b$ increases. The second candidate of the estimation became a complex number when $b>0.5$. Concerning the starting delay, it appears that $b=0.0 \sim 0.2$ is acceptable. As a conclusion, it is preferable that the signal for the real number Laplace method identification are modified by considering the following remarks.

- The drift and offset are removed from the output signal so as to make the signal zero at the input of zero.

- Both the input signal and the output signal include only positive values; in short, these signals do not contain zero-crossing.

- Identification is started from just after the rising up of the input signal. 

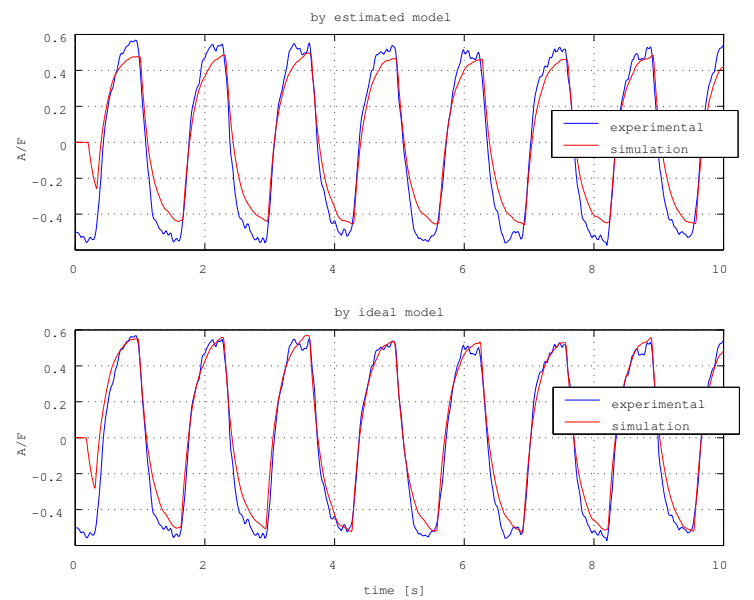

Fig. 6. Simulated responses computed by the identified model (upper) and by the ideal model (lower)

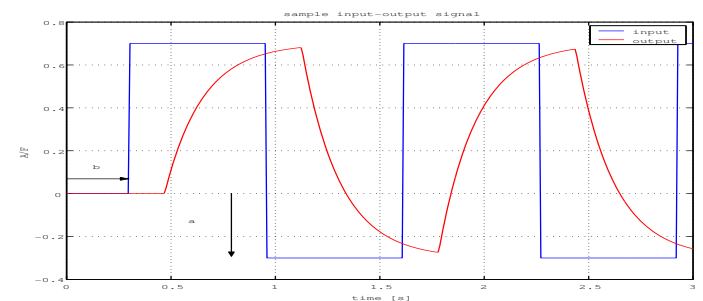

Fig. 7. Test signal for evaluation

\section{Discussion}

As the real number Laplace method includes a numerical integral computation, this method appears seemingly to require much memory at the implementation, but it is not true. This worry will be removed by the following two artifices.

The first artifice is a preliminary computation of the constant terms of the equations. Generally, in case of an on-line identification methods based on the least-square computation, the regressor vector includes time-varying variables that come from the measured signals; hence, it is necessary to compute them on-line. And as shown in Eq. (12), the inverse matrix computation of $\left(\Phi^{T} \Phi\right)^{-1}$ is included. Thus, the normal least-square-based method requires an on-line inverse matrix computation. This computation requires a high level of the arithmetic operation; hence, it is not welcomed for the computer device of a consumerlevel product. Meanwhile, in case of the real number Laplace method, $\Phi$ is a constant matrix as $\Phi:=\left[\varphi\left(\sigma_{1}\right)^{T}: \varphi\left(\sigma_{M}\right)^{T}\right]^{T}$, where $\varphi\left(\sigma_{i}\right)$ is a constant vector given by Eq. (7), and this computation can be finished offline. Thus, troublesome inverse matrix computation can be replaced by simple summation and multiplication. 


\begin{tabular}{|c|r|rr|rr|}
\hline$a$ & $\hat{K}$ & $\hat{T}_{a}$ & $\hat{T}_{b}$ & $\hat{L}_{a}$ & $\hat{L}_{b}$ \\
\hline 0 & 1.000 & 0.176 & 0.155 & 0.174 & 0.195 \\
0.1 & 1.000 & 0.180 & 0.170 & 0.170 & 0.181 \\
0.2 & 1.000 & 0.187 & 0.188 & 0.165 & 0.163 \\
0.3 & 1.000 & 0.197 & 0.215 & 0.155 & 0.138 \\
0.4 & 1.000 & 0.219 & 0.257 & 0.136 & 0.099 \\
0.5 & 1.000 & 0.288 & 0.353 & 0.078 & 0.014 \\
0.6 & 16.91 & 7.217 & 3.559 & 8.042 & 11.70 \\
\hline true & $K=1.0$ & $T=0.18$ & & $L=0.17$ & \\
\hline
\end{tabular}

Table 7. Result of identifications when a ratio of the positive-value zone of an input signal was changed

\begin{tabular}{|c|r|rr|rr|}
\hline$b$ & $\hat{K}$ & $\hat{T}_{a}$ & $\hat{T}_{b}$ & $\hat{L}_{a}$ & $\hat{L}_{b}$ \\
\hline 0 & 1.000 & 0.176 & 0.155 & 0.174 & 0.195 \\
0.1 & 1.000 & 0.175 & 0.154 & 0.174 & 0.195 \\
0.2 & 1.000 & 0.174 & 0.147 & 0.176 & 0.202 \\
0.3 & 1.000 & 0.170 & 0.130 & 0.179 & 0.219 \\
0.4 & 1.000 & 0.163 & 0.080 & 0.185 & 0.268 \\
0.5 & 1.000 & 0.151 & c.n. & 0.196 & c.n. \\
0.6 & 1.000 & 0.132 & c.n. & 0.213 & c.n. \\
\hline true & $K=1.0$ & $T=0.18$ & & $L=0.17$ & \\
\hline
\end{tabular}

Table 8. Result of identification against different starting delay

The second artifice is an on-line accumulation of the input/output data instead of the original integral computations. For the implementation to a computer, $2 M$ buffer memory $\tilde{U}\left(\sigma_{1}\right) \sim$ $\tilde{U}\left(\sigma_{M}\right)$ and $\tilde{Y}\left(\sigma_{1}\right) \sim \tilde{Y}\left(\sigma_{M}\right)$ for $\sigma_{1} \sim \sigma_{M}$ are prepared, then recurrence equations:

$$
\begin{aligned}
\tilde{U}\left(\sigma_{1}\right) & =\tilde{U}\left(\sigma_{1}\right)+u[i] \cdot e^{-\sigma_{1} \Delta \cdot i} \cdot \Delta \\
& \vdots \\
\tilde{U}\left(\sigma_{M}\right) & =\tilde{U}\left(\sigma_{M}\right)+u[i] \cdot e^{-\sigma_{M} \Delta \cdot i} \cdot \Delta \\
\tilde{Y}\left(\sigma_{1}\right) & =\tilde{Y}\left(\sigma_{1}\right)+y[i] \cdot e^{-\sigma_{1} \Delta \cdot i} \cdot \Delta \\
& \vdots \\
\tilde{Y}\left(\sigma_{M}\right) & =\tilde{Y}\left(\sigma_{M}\right)+y[i] \cdot e^{-\sigma_{M} \Delta \cdot i} \cdot \Delta
\end{aligned}
$$

are used for computing Eqs. (13) and (14). This technique is useful to reduce memory on the computer architecture.

\section{Conclusion}

In this chapter, a practical, accurate yet simple identification method, termed as real number Laplace method, to estimate parameters of a first-order system including a time-delay was proposed. The key point is restriction of a domain of the Laplace transformation to real numbers. 
The method can estimate the system parameters and the time-delay simultaneously on-line. The method does not require a priori information about the time-delay, and can be applied to a system including arbitrary time-delay. Comparison with other method (based on recursive least-square identification with a bilinear transformed impulse transfer model) proved that the real number Laplace method could identify the system parameters much accurately. And a relation between an accuracy of the identified results and the tuning conditions were investigated, and a guideline for the tuning was summarized. Moreover, the real number Laplace method was applied to an actual response data of an engine sensor, and the sensor dynamics and the unknown time-delay could be estimated with sufficient accuracy. Further, a guideline of a preliminary modification of the measured data was shown, and remarks for better identification on the actual system were summarized.

\section{A. Recursive least-square identification using an impulse transfer function with a bilinear transformation}

To a first-order system with a time-constant $T$ and a gain $k$ :

$$
G(s)=\frac{k}{1+T s}
$$

applying the bilinear transformation:

$$
s=\frac{2}{\Delta} \frac{1-z^{-1}}{1+z^{-1}}
$$

yields the impulse transfer function as

$$
\begin{aligned}
G(z)= & \frac{\Delta k \cdot z^{-1}+\Delta k}{(\Delta-2 T) \cdot z^{-1}+(\Delta+2 T)} \\
=: & \frac{z^{-1}+1}{b_{2} z^{-1}+b_{1}} \\
& b_{2}:=\frac{\Delta-2 T}{\Delta k} \\
& b_{1}:=\frac{\Delta+2 T}{\Delta k},
\end{aligned}
$$

where $z^{-1}$ is a time-shift operator. $T$ and $k$ can be derived from Eqs. (26) and (27) as

$$
\begin{aligned}
T & =\frac{b_{1}-b_{2}}{b_{1}+b_{2}} \cdot \frac{\Delta}{2} \\
k & =\frac{2}{b_{1}+b_{2}} .
\end{aligned}
$$

Once $b_{1}$ and $b_{2}$ are obtained, $T$ and $k$ can be computed from Eqs. (28) and (29).

Next, the measured input and output data are described as $\bar{u}(t)$ and $\bar{y}(t)$ respectively, and the inverse Z-transformation is applied to Eq. (25), then

$$
b_{2} \cdot \bar{y}(t-1)+b_{1} \cdot \bar{y}(t)=\bar{u}(t-1)+\bar{u}(t)
$$


is obtained. Furthermore, Eq. (30) is transformed as

$$
\left[\begin{array}{c}
\bar{u}(t)+\bar{u}(t-1) \\
\bar{u}(t-1)+\bar{u}(t-2) \\
\cdots \\
\bar{u}(2)+\bar{u}(1)
\end{array}\right]=\left[\begin{array}{cc}
\bar{y}(t) & \bar{y}(t-1) \\
\bar{y}(t-1) & \bar{y}(t-2) \\
\ldots & \ldots \\
\bar{y}(2) & \bar{y}(1)
\end{array}\right]\left[\begin{array}{l}
b_{1} \\
b_{2}
\end{array}\right] .
$$

Using replacements as

$$
\begin{aligned}
y(t) & =\bar{u}(t)+\bar{u}(t-1) \\
\varphi(t) & =[\bar{y}(t), \bar{y}(t-1)]^{T}
\end{aligned}
$$

Eq. (31) is transformed as

$$
\begin{aligned}
{\left[\begin{array}{c}
y(t) \\
y(t-1) \\
\ldots \\
y(2)
\end{array}\right] } & =\left[\begin{array}{c}
\varphi^{T}(t) \\
\varphi^{T}(t-1) \\
\vdots \\
\varphi^{T}(2)
\end{array}\right]\left[\begin{array}{l}
b_{1} \\
b_{2}
\end{array}\right] \\
\Rightarrow \mathbf{y} & =\Phi \cdot \theta .
\end{aligned}
$$

Last step is an estimation of vector term $\Phi$ that includes parameters $b_{1}$ and $b_{2}$, and is executed by the recursive least-square method. Finally, $T(t)$ and $k(t)$ can be computed from Eqs. (28) and (29) on-line.

\section{References}

[1] R. C. Miall, D. J. Weir, D. M. Wolpert and J. F. Stein. Is the Cerebellum a Smith Predictor? Journal of Motor Behavior, 25(3):203-216, 1993.

[2] O. J. M. Smith. A Controller to Overcome Dead Time. Transaction ISA, 6(2):28-33, 1959.

[3] P. V. D. Hof and R. J. P. Schrame. Identification and Control - Closed-loop Issues. Automatica, 31(12):1751-1770, 1995.

[4] X. Li and C. E. de Souza. Delay-dependent Robust Stability and Stabilization of Uncertain Linear Delay Systems: A Linear Matrix Inequality Approach. IEEE Transaction on Automatic Control, 42(8):1144-1148, 1997.

[5] P. Albertos and A. Sala. Iterative Identification and Control. Springer, 2002.

[6] F. Reed, P. Feintuch, and N. Bershad. Time-delay estimation using the LMS adaptive filter-static behavior. IEEE Trans. Acoustics, Speech, and Signal Processing, 29(3):561-576, 1981.

[7] F. C. Teng, and H. R. Sirisena. Self-tuning PID controllers for dead time process. IEEE Trans. Industrial Electronics, 35(1):119-125, 1988.

[8] A. B. Bulsari (ed.). Neural network for Chemical Engineering. Elsevier Science, Amsterdam, Holland, 1995.

[9] R. Yang, X. Xu, and C. Zhang. Delay-dependent robust $H_{\infty}$ filtering for uncertain state delayed system. in Proc. of the IFAC 15th Triennial World Congress, in CD-ROM, Barcelona, Spain, 2002.

[10] Y. Tan, C.-Y. Su, and N. Karim. Neural network based time-delay estimation for nonlinear dynamic systems. in Proc. of the IFAC 15th Triennial World Congress, in CD-ROM, Barcelona, Spain, 2002. 
[11] T. Hamada, and K. Nakano. Wavelet-based Underdetermined Blind Source Separation of Speech Mixtures. in Proc. of the ICCAS 2007, in CD-ROM, Seoul, Korea, 2007.

[12] L. Guzzella and C. H. Onder. Introduction to Modeling and Control of Internal Combustion Engine Systems. Springer-Verlag, Berlin, 2004.

[13] S. Suzuki, and K. Furuta. Real Number Laplace Transformation-based Identification for First-order System including Time-delay, in Proc. of the 2008 IEEE International Conference on Emerging Technologies and Factory Automation (ETFA2008), Hamburg, Germany; 143$149,2008$. 


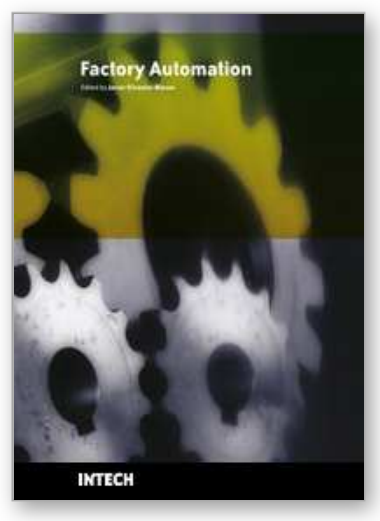

\author{
Factory Automation \\ Edited by Javier Silvestre-Blanes
}

ISBN 978-953-307-024-7

Hard cover, 602 pages

Publisher InTech

Published online 01, March, 2010

Published in print edition March, 2010

Factory automation has evolved significantly in the last few decades, and is today a complex, interdisciplinary, scientific area. In this book a selection of papers on topics related to factory automation is presented, covering a broad spectrum, so that the reader may become familiar with the various fields, and also study them in more depth where required. Within various chapters in this book, special attention is given to distributed applications and their use of networks, since it is one of the most relevant subjects in the evolution of factory automation. Different Medium Access Control and networks are analyzed, while Ethernet and Wireless networks are looked at in more detail, since they are among the hottest topics in recent research. Another important subject is everything concerning the increase in the complexity of factory automation, and the need for flexibility and interoperability. Finally the use of multi-agent systems, advanced control, formal methods, or the application in this field of RFID, are additional examples of the ideas and disciplines that experts around the world have analyzed in their work.

\title{
How to reference
}

In order to correctly reference this scholarly work, feel free to copy and paste the following:

Satoshi Suzuki and Katsuhisa Furuta (2010). Easy-Implementable On-line Identification Method for a FirstOrder System Including a Time-Delay, Factory Automation, Javier Silvestre-Blanes (Ed.), ISBN: 978-953-307024-7, InTech, Available from: http://www.intechopen.com/books/factory-automation/easy-implementable-online-identification-method-for-a-first-order-system-including-a-time-delay

\section{INTECH}

open science | open minds

\section{InTech Europe}

University Campus STeP Ri

Slavka Krautzeka 83/A

51000 Rijeka, Croatia

Phone: +385 (51) 770447

Fax: +385 (51) 686166

www.intechopen.com

\section{InTech China}

Unit 405, Office Block, Hotel Equatorial Shanghai

No.65, Yan An Road (West), Shanghai, 200040, China

中国上海市延安西路65号上海国际贵都大饭店办公楼405单元

Phone: +86-21-62489820

Fax: +86-21-62489821 
(C) 2010 The Author(s). Licensee IntechOpen. This chapter is distributed under the terms of the Creative Commons Attribution-NonCommercialShareAlike-3.0 License, which permits use, distribution and reproduction for non-commercial purposes, provided the original is properly cited and derivative works building on this content are distributed under the same license. 